\title{
Editorial
}

\section{The Evolution of Laser in ENT}

\section{Introduction}

It has been said that when the time in which we live is finally names, it will not be known as the atomic or space age, but the laser age. The laser is not universally accepted as technologically the most advanced tool in various specialties of surgery. However the properties of all lasers in not alike and the field of Ear, Nose and Throat surgery has advanced significantly with the introduction of the various lasers. Laser is especially studied for ENT work and finds application in various aspects of surgery in the ear, nose larynx, neck etc., the quality of ENT surgery using laser improves significantly on the results. Lasers have revolutionized the way otolarnygologists approach surgery, enabling highly precise and, often, less invasive treatment of a wide range of ENT applications

\section{The Discovery of Laser}

Albert Einstein propelled the theoretical foundation for the development of this landmark invention in 1916 when he proposed that photons could stimulate emission of identical photons from excited atoms. ${ }^{1}$ Stimulated emission is where a photon interacts with an excited molecule or atom and causes the emission of a second photon having the same frequency, phase, polarization and direction. This theory has been fundamental in the development of laser technology. In 1940, the Russian physicist Valentin Fabrikant proposed that stimulated emission in a gas discharge may amplify light under specific conditions ${ }^{1}$. However, it was not until after the end of World War II when research into laser technology benefitted greatly from corporate and government investments into technological progress during the cold war era. This coupled with the growth in numbers of physicists and engineers and growth in the economy created the foundations for the invention of the ruby laser in 1960 by Theodore Maiman². Soon after Maiman built the first laser, his assistant joked that the laser was "a solution looking for a problem." This of course contained some truth to it, as the laser was not a device invented to fill specific application requirement. It was more a discovery than an invention,

\section{The Principles of Laser Surgery}

The atoms of a laser exist within a medium which can be either solid, liquid or gas. This is encased within an optical resonant chamber in-between two mirrors. The process of laser emission begins with an external source of energy, such as a flash lamp or an electric arc, which is used to excite the atoms in the medium. The internal energy contributes to a cascade of stimulated emissions that create the amplified light energy. The three important properties to laser are the wavelength, coherence and directionality. The particular wavelength of laser energy will decide it's precision as a scalpel and the haemostatic properties. The laser energy must be intense, sharply focused, and absorbed almost entirely at the surface for precise dissection with minimal surrounding thermal injury and tissue damage. 1,3,4

\section{Lasers available}

Currently there are a large number of lasers available for the otolaryngologist to utilise. 
They have varying characteristics including different wavelengths, interactions with tissue, modes of transmission and delivery systems. These include the Carbon Dioxide (CO2), Diode, neodymium: yttrium-aluminum-garnet (Nd:YAG) in contact and non-contact modes, argon, potassium titanyl phosphate (KTP), Pulsed Dyed Laser (PDL), argon dye, holmium:YAG, erbium:YAG and free electron lasers. ${ }^{3,5,6}$

\section{Tissue Interactions}

Laser light falling of tissues may be reflected, scattered, transmitted Or absorbed. Only the absorbed light causes a tissue reaction. The main substance absorbing the laser' is called the primary chromophore. Absorption produces mainly kinetic excitation of the absorbing molecules. Kinectic excitation produces thermal effects ranging from reversible hyperthermia through enzyme deactivation, protein denaturization and coagulation to dehydration, vaporization and carbonization.

\section{Why are lasers used?}

Lasers are used for many reasons. In many instances, they can improve the precision of the surgeon with their finely focused beams of light. Some lasers can reduce bleeding by coagulating blood vessels as they cut tissue. Others can be aimed down narrow passages or sent down fiber-optic channels in endoscopes to reach areas that are otherwise inaccessible.

A new trend in laser surgery is to use the special properties of different wavelengths of laser light to selectively treat different problems. For instance, a yellow laser light absorbed by the red blood cells of a birthmark called a "portwine stain" can result in the selective destruction of the birthmark without affecting the skin cells around it. The result is that scarring of the remaining normal skin is avoided. In another instance of selective laser treatment, drugs that are retained by tumors are infused into the body.
In many instances, the cost of medical care can be reduced by lasers. Through their use, many procedures that previously required hospital admission can be done on an outpatient basis. In some instances, they can reduce pain, enabling a quicker return to work. It is important to discuss the pros and cons of laser use in your particular instance with your doctor.

and activated by lasers to destroy only the tumor, preserving normal structures.

\section{Uses in ENT}

Further to its growing use in otolaryngology, laser surgery has developed in other areas of otolaryngology. Laryngology is one of the specialty areas in which lasers are most often used. The $\mathrm{CO} 2$

laser is by far the laser of choice. Because of the precise cutting and superficial well delineated effect of the $\mathrm{CO} 2$ laser, it is widely used in laryngology for delicate phonatory surgery like vocal cord polyp, nodule, arytenoids granuloma, web, synachia etc, precise excision of carcinoma in situ or early (T1) tumors, and vaporization of bulky obstructing carcinoma of the upper airway. Tumours of the tongue, floor of the mouth, palate and tonsils can be resected with excellent control and haemostasis using the $\mathrm{CO} 2$ laser. The Nd:YAG, CO2, KTP, and diode lasers have all been used with good short-term results of nasal airway obstruction and recurrent epistaxis. In ear surgery the precision of argon, KTP, and CO2 lasers through a microscope have been shown to be very effective in stapedotomy, ossicular fixation and tympanosclerosis. Diode laser has been increasingly used in endo nasal Dacryocystorhinostomy. It is less invasive because bony and mucosal part is opened and widened with precision using diode laser.

\section{Advantages and limitations}

Laser surgery has advantages when compared to cold surgery on the larynx. It offers an unobstructed view of the operation 
field, ability to work at longer distances, minimises tissue manipulation, increased sterility, better haemostasis and is associated with fewer post operative complications. However, lasers require a large number of personnel to ensure effectiveness and safety. There is also a significant cost associated with the installation of equipment, maintenance and updating technical developments can be significantly more costly than using cold instruments. Laser heat can increase scarring and cause damage to adjacent tissue. Other potential limitations of laser include potential for endotracheal explosion, facial burns, mucosal burns, vocal fold webs, stenoses and glottic incompetence ${ }^{3-5}$

\section{The Future}

Years of development have vastly improved laser performance and have been an important solution for a variety of problems, not only in laryngology, but also in scientific research, consumer products, telecommunications, engineering and a host of other applications.

In the treatment of laryngeal cancer, laser surgery has already demonstrated reliable tumour removal with fewer complications than open surgery in managing many glottic and supraglottic lesions. Currently, there are a variety of different lasers available for otolaryngologists to utilise with extended applications and variable wavelengths such as CO2, Nd:YAG, argon, PDL, KTP and argon dye. New lasers, such as the use of thulium on endolaryngeal resections, show promising results offering improved haemostasis and effective tangential dissection. The increased volume of ambulatory and office-based laser surgery has an important role in reducing costs and will continue to gain increased interest in financially stretched healthcare systems around the world. The considerable amount of research that continues to develop the use of laser in otolaryngology inevitably means further advancements in its clinical application ${ }^{3,4}$

\section{Conclusion}

The existing uses for lasers in Otolaryngology are constantly being refined, and new applications of the technology are constantly being developed. Lasers are being developed to produce light at every wavelength, and the development of tunable laser systems means one machine could be used for all wavelengths. Improved light delivery systems are being developed, and new photosensitizers are being produced with more desirable characteristics and fewer side effects. The considerable amount of effort going into research in this field means further advances in the clinical application of laser technology are to be expected.

\section{Professor Dr. Md. Rojibul Haque}

National Institute of ENT

Tejgaon, Dhaka.

E-mail: rojibul@yahoo.com

\section{References}

1. Hecht JA. Short History of Laser Development. Applied Optics 2010; 49: 99-122.

2. Maiman TH. Stimulated Optical Radiation in Ruby. Nature 1960;187: 493-494

3. Zeitels SM. and Burns, JA. Laser Applications in Laryngology: Past, Present, and Future. Otolaryngologic Clinics of North America 2006; 39: 159-172.

4. Yan $\mathrm{Y}$ et al. Use of Lasers in Laryngeal Surgery. Journal of Voice 2010; 24: 102109.

5. Shapiro J, Zeitels, SM. and Fried MP. Laser Surgery for Laryngeal Cancer. Operative Techniques in OtolaryngologyHead and Neck Surgery2008; 3: 84-92

6. Ossoff $\mathrm{RH}$, et al. Clinical Applications of Lasers in Otolaryngology-Head and Neck Surgery. Lasers in Surgery and Medicine 2009; 15: 217-248. 